\title{
Stereotypen geht es gut. Ein Beitrag zu stereotypischem Denken polnischer und deutscher Studierender am Beispiel eines E-Mail-Tandemprojekts
}

\section{Stereotypes are well established. On stereotypical thinking from the perspective of Polish and German students based on an e-mail tandem project}

\begin{abstract}
The article attempts to approach stereotypes from both the theoretical and empirical perspectives. First, it endeavours to explain the term "stereotype", looks into its characteristics and functions and then presents selected aspects of the Polish-German tandem project devoted to stereotypical thinking.

KEYWORDS: autostereotype, heterostereotype, German as a foreign language, stereotype, tandem language learning.

SCHLÜSSELWORTE: Autostereotyp, Deutsch als Fremdsprache (DaF), Heterostereotyp, Stereotyp, Sprachenlernen im Tandem.
\end{abstract}

\section{VORBEMERKUNG}

Im Grunde treten Stereotypen in allen sozialen Bereichen auf - es gibt professionelle Stereotypen (der zerstreute Professor, der weltfremde Wissenschaftler, der ,Pauker', der windige Versicherungsvertreter, der hektische oder arrogante Manager), konfessionelle bzw. religiöse Stereotypen (der fanatische - oder heute 
fundamentalistische - Mohammedaner, der dogmatische Katholik), Klassenstereotypen (der ausbeuterische Bourgeois, der Prolet, der beschränkte Kleinbürger) oder auch sexistische Stereotypen (die Frau am Steuer). Viele Komödien, ob im Theater oder im Film, leben von der Existenz und allgemeinen Akzeptanz solcher Stereotypen. Als besonders wirksam und verbreitet haben sich nationale bzw. ethnische und, innerhalb einer Nation, regionale Stereotypen erwiesen: sie sind das Lieblingsobjekt der Stereotypen-Forschung fast aller Wissenschaftsdisziplinen (Hahn \& Hahn 2002: 19).

Die Allgegenwart von Stereotypen lässt sich kaum bestreiten. Schließlich begegnet man ihnen sowohl im Privaten als auch im Beruflichen, im In- und Ausland. Kein Lebensbereich scheint von ihnen verschont zu bleiben. Auch im fremdsprachlichen Unterricht gehören sie dazu1. Denn, wie Bechtel (2003: 77) mit Recht hervorhebt, bestehe eine seiner Aufgaben darin, den Lernenden trotz knapper Zeit und begrenzter Sprachkenntnisse eine Vorstellung von der Zielkultur bzw. von den Sprechern der Zielkultur zu vermitteln. Daher müsse dieser verallgemeinern. Zum anderen trägt jeder Fremdsprachenlehrer oder -lerner ${ }^{2}$ bereits bestimmte Vorstellungen von der fremden Kultur in den Unterricht hinein.

Bedeutet dies etwa, dass der Abbau von Stereotypen ein sinnloser, zum Scheitern verurteilter Kampf mit einem immer leicht vorauszusehenden Ergebnis ist, hinter dem sich eine utopische Hoffnung auf eine tolerante Welt verbirgt? Oder ist die Niederlage in diesem Ringen um mehr Verständnis Anderen gegenüber doch gar nicht vorprogrammiert?

Im vorliegenden Beitrag sollen Stereotype aus dem theoretischen und empirischen Blickwinkel beleuchtet werden. Deswegen werden das Wesen, Merkmale und auch Funktionen stereotypen Denkens näher präsentiert, um im Nachhinein den Versuch zu wagen, sich mit Stereotypen vor dem Hintergrund eines polnisch-deutschen E-Mail-Tandemprojekts auseinanderzusetzen. An diesem Beispiel wird versucht, zu zeigen, wie im fremdsprachlichen Unterricht mit Stereotypen umgegangen werden und was diese Auseinandersetzung bringen kann.

\section{DAS STEREOTYP ALS EIN KOMPLEXES SOZIALES PHÄNOMEN}

Will man die wissenschaftliche Diskussion über ein Phänomen in die Wege leiten, ist es zunächst unumgänglich, es näher zu charakterisieren. Daher wird im vorliegenden Beitrag zunächst der Terminus Stereotyp erläutert, um im nächsten Schritt auf seine Merkmale und Rolle einzugehen.

\footnotetext{
${ }^{1}$ Mit Stereotypen im Fremdsprachenunterricht hat sich bereits Keller (1969) auseinandergesetzt.

${ }^{2}$ Maskuline Personenbezeichnungen werden in dem Beitrag im generischen Sinne verstanden.
} 


\subsection{Stereotyp als...?}

Der Begriff Stereotyp ist auf zwei griechische Wörter zurückzuführen: ,stereós' steht für ,fest, haltbar, hart, starr', während, týpos' etwa ,artig' bedeutet. Zum ersten Mal wurde er nachweislich 1798 vom französischen Drucker Didot zur Bezeichnung eines neuen Druckverfahrens eingesetzt:

Das Stereotyp bildet das mittels einer Matrize hergestellte Duplikat einer Druckform, eines Druckstocks. Als fester Drucksatz ermöglicht es eine fast unbegrenzte Vervielfältigung von Zeitungsseiten, aber auch von schon bestehenden Büchern, Schriften sowie deren Aufbewahrung für spätere, erneute und unveränderte Auflagen und Veröffentlichungen (Koch 2002: 266-267).

Im Laufe der Jahrhunderte kam allerdings eine neue Auslegung des Terminus hinzu, auf die in zahlreichen Wissenschaftsdisziplinen zurückgegriffen wird. Der amerikanische Publizist Walter Lippmann zog 1922 den Begriff Stereotyp aus der Druckereiwelt heran, um sich mit ihm aus der sozialwissenschaftlichen Perspektive auseinanderzusetzen. Den Ausgangspunkt seiner Überlegungen bildet die kognitive Grundlage der Stereotypisierung. Zwar hebt er hervor, dass Motivation und sozialer Kontext der äußeren Welt einen nicht geringen Einfluss auf das Stereotyp nehmen könnten, aber zugleich unterstreicht er, dass die "Bilder in unserem Kopf“ („pictures in our head") mit der Wirklichkeit gar nicht übereinstimmen müssten. Sie würden jedoch das menschliche Verhalten mehr als die objektiven Bedingungen der jeweiligen Situation steuern (Konrad 2006: 15-16). Zum Stereotyp konstatiert Lippmann Folgendes:

Sein kennzeichnendes Merkmal besteht darin, daß es der Anwendung der Vernunft vorausgeht, es ist eine Form der Wahrnehmung, prägt den Mitteilungen unserer Sinne einen bestimmten Charakter auf, bevor diese Mitteilungen unsere Vernunft erreichen (Schaff 1980: 41).

Eine heutzutage nicht weniger verbreitete, obschon aus der Sicht der Sprachwissenschaft formulierte, Definition von Stereotyp hat Ute Quasthoff (1973) vorgeschlagen:

Ein Stereotyp ist der verbale Ausdruck einer auf soziale Gruppen oder einzelne Personen als deren Mitglieder gerichteten Überzeugung. Es hat die logische Form eines Urteils, das in unberechtigt vereinfachender und generalisierender Weise, mit emotional-wertender Tendenz, einer Klasse von Personen bestimmte Eigenschaften oder Verhaltensweisen zu- oder abspricht (Quasthoff 1973: 28).

In den beiden bereits präsentierten Definitionen wird der subjektive Charakter von Stereotypen zum Ausdruck gebracht, der meist wenig - wenn überhaupt - rational begründbar ist. 
Einen Definitionsvorschlag, der mit den oben aufgegriffenen Erläuterungen eine gewisse Ähnlichkeit aufweist, macht Pfeiffer (2000: 127), für den Stereotype durch die Übertragung von Bildern durch Drittpersonen bzw. äußere Einflüsse entstehen und somit nicht auf der eigenen Erfahrung bauen. Stereotype als mentale Bilder spiegeln dementsprechend die Wirklichkeit nicht in ihrer Komplexität wider, sondern präsentieren sie einfach strukturiert und zugleich inadäquat. Der Forscher hebt dabei hervor, dass es mit zunehmendem Alter immer schwieriger werde, die einmal entworfenen Bilder zu hinterfragen. Aus diesem Grund würden Menschen mit einem festgefügten Bild über eine Person bzw. einen Sachverhalt alle weiteren Informationen über diese bzw. diesen nur insofern aufnehmen, als dadurch ihr bereits bestehender Blickpunkt, d.h. ihr bereits gefügtes Bild bestätigt werde. Dieser Prozess selektiver Wahrnehmung lasse Menschen Widersprüche vermeiden und ihr Selbstbild legitimieren.

Auf den vereinfachenden Charakter von Stereotypen weisen auch Erll und Gymnich (2007: 72-73) hin und betrachten diese als „[...] reduktionistische Ordnungsraster, die sich oft in formelhaften Wendungen und Gemeinplätzen äußern (der, fleißige Deutsche', der, perfide Engländer', der ,lebensfreudige Franzose', etc.)“. Die beiden Forscher ordnen Stereotype dem Bereich des erstarrten Denkens zu. Denn es sind festgefahrene Schemata, die einem häufig gar nicht bewusst sind.

Für Six (1987: 44), einen der Vertreter der kognitiven Sozialpsychologie3, gelten Stereotype wiederum als ,[...] spezifische Wahrnehmungs- und Urteilsmuster von Personen und Gruppen, ein wichtiges Konzept im Themengebiet der sozialen Urteilsbildung oder der Personenwahrnehmung".

Bereits die oben vorgenommenen Ausführungen zum Terminologischen verdeutlichen die Mehrdimensionalität von Stereotypen. Der Begriff erfreut sich beispielsweise in der Ethnologie, Fremdsprachendidaktik, Linguistik, Politologie, Psychologie oder Soziologie eines nicht nachlassenden Interesses und wird jeweils aus einem anderen Blickwinkel beleuchtet. Auch im Alltag scheinen alle $\mathrm{zu}$ wissen, was unter stereotypem Denken $\mathrm{zu}$ verstehen ist. Gerade die Tatsache, dass der Terminus in so vielen Bereichen vertreten ist, macht seine definitorischen Schwierigkeiten aus.

${ }^{3}$ Vertreter der kognitiven Sozialpsychologie waren diejenigen, die ein neutrales Stereotypenkonzept hauptsächlich verbreitet haben. In dieser Disziplin werden Stereotype von der Vorurteilsforschung getrennt und separat behandelt (Dąbrowska 1999: 98). Dementsprechend versteht O’Sullivan (1992: 65, nachfolgend Marques-Schäfer, Bolacio Filho \& Stanke 2016: 569) Stereotype eher als eine gefühlsmäßig neutrale, kognitive Form der Verallgemeinerung. Vorurteile sind für den Forscher dagegen stärker mit Gefühlen besetzt, können eher zu einem diskriminierenden Verhalten führen und demzufolge eine von vornherein feindselige Haltung ausdrücken. 


\subsection{Merkmale von Stereotypen}

In den oben präsentierten Definitionen wurden - wenn auch meist implizit - unterschiedliche Merkmale von Stereotypen aufgegriffen, auf die im weiteren Teil ausführlicher eingegangen wird.

Unterzieht man die in Unterkapitel 2.1 präsentierten Definitionen einer Analyse im Hinblick auf Merkmale, ergibt sich bereits auf den ersten Blick ein Bild von einem schwer revidierbaren oder sogar - um einen Schritt weiter zu wagen - gegen jegliche Änderungen resistenten Phänomen, das sich aus den Aussagen Dritter speist.

Eine umfangreiche Charakteristik von Stereotypen liefern Berting und Villain-Gandossi (1999: 14-15):

- Stereotype stellen keine allgemeine Meinungsäußerung dar, sondern sie sind eine mehr oder weniger allgemeine Widerspiegelung gesellschaftlicher Phänomene. Des Öfteren stehen sie mit sprachlichen Topoi (d.h. mit Lexemen oder Lexemzusammensetzungen) in engem $\mathrm{Zu}$ sammenhang und ihre Verbalisierung zieht eine Hülle nebelhafter Assoziationen und Konnotationen nach sich. Darüber hinaus haben diese Abbilder immer wertenden Charakter.

- Sie entspringen nicht unmittelbaren Erfahrungen des Menschen, sondern der Tradition, der Überlieferung und der Vermittlung. Da sie oft im kollektiven Bewusstsein fest verankert sind, sind sie für Erfahrungen unauffällig, die nicht diesen fixen Vorstellungen gerecht werden.

- Stereotype gelten als ein wichtiges, gesellschaftlich integrierendes Element, das in die Motivation sozialen Agierens, in die Ideologie oder in die politische Propaganda Eingang finden kann.

- Stereotype sind positive oder negative Urteile, die auf Überzeugung bauen.

- Stereotype entstehen in der Gesellschaft und werden dem Individuum als Ausdruck der öffentlichen Meinung durch die Familie sowie - im weitesten Sinne - durch das gesellschaftliche Umfeld ohne Rücksicht auf dessen individuelle Erfahrungen übermittelt.

- Stereotype sind (positiv oder negativ) emotional geladen.

- Sie können dem Ist-Zustand vollständig oder teilweise widersprechen.

- Stereotype bleiben über lange Zeit gegen jegliche Änderungen immun, da sie von den tatsächlichen Erfahrungen von Menschen weitgehend unabhängig sind.

In der präsentierten Auflistung wird insbesondere eine starke Gebundenheit von Stereotypen an die jeweilige Gesellschaft hervorgehoben. Somit ist stereotypes Denken nicht ein vom Individuum abhängiges Phänomen, sondern es funktioniert prinzipiell in der kollektiven Dimension, sozusagen als 
„Erbe“ der jeweiligen Sprach- und Kulturgemeinschaft. Demzufolge erfüllt es in dieser Hinsicht zahlreiche, für eine Gemeinschaft relevante Funktionen, die unten thematisiert werden. Auf diesen Aspekt macht Bartmiński (1999: 313) aufmerksam:

Sie [Stereotype] sind ein wesentlicher Bestandteil des sprachlichen Weltbildes und des kulturellen Kodes. Da sie obligatorisch mit der natürlichen Sprache verbunden sind, sind sie so, wie unsere Alltagssprache: gegenständlich beschreibend und subjektiv wertend, akzeptierend und kritisierend, eingebunden in historische Erfahrungen, in ererbte mythologische Traditionen einzelner Gruppen, und zugleich den momentanen Absichten der Sprechenden unterworfen. Als Produkte psychologischer und soziokultureller Mechanismen lassen sie sich nicht beseitigen, sondern nur in gewissen Grenzen bewusst gestalten.

Stereotype gelten somit - wenn auch nur in gewissem Grad - als Bestandteil des Sozialisationsprozesses, da sie - von den Vertretern der jeweiligen Sprach- und Kulturgesellschaft getragen - auf ihre neuen Mitglieder in größerem oder geringerem Maße stets übertragen werden.

Auch für Telus (2002: 66-67) steht die soziale Ausrichtung von Stereotypen im Vordergrund. Denn ihren Gegenstand bilden in erster Linie bestimmte Gruppen von Menschen (rassische, nationale, klassenmäßige, politische, berufliche Gruppen genauso wie Geschlechtsgruppen, etc.) sowie sekundär die zwischen ihnen bestehenden Beziehungen. Ihre Genese ist sozial, d.h. sie werden dem Individuum als Ausdruck der öffentlichen Meinung durch Erziehung seitens der Familie oder des Milieus vermittelt, und zwar unabhängig von seiner persönlichen Erfahrung. Dank den bereits genannten Merkmalen können die Stereotype ihrer sozialen Funktion gerecht werden. Diese besteht darin, die von der jeweiligen Gesellschaft oder Gruppe akzeptierten Werte und Urteile zu verteidigen, deren Internalisierung als verbindliche soziale Norm Voraussetzung für die Integrierung des Einzelnen in die Gruppe ist.

Für Hahn und Hahn (2002: 22) prägt sich jeder Mensch die gespeicherten Kenntnisse und Erfahrungen seiner Gesellschaft samt einem das soziale Leben regulierenden Wertesystem ein. Begriffe gelten dabei als verbaler Ausdruck, wie solche notwendigen Verallgemeinerungen gelernt und benutzt werden. Während sich Begriffe auf Erfahrungen stützen, eine kognitive Funktion - wenn auch mit emotionaler Färbung - und eine bestimmte Beziehung zur Realität haben, sind Stereotype Generalisierungen mit einer emotional stark ausgeprägten Komponente. Sie werden durch die Umgebung affektiv vermittelt. Begriffe weisen eine starke Gebundenheit an die Realität auf, d.h. ihr Informationsgehalt ist überprüfbar, verifizierbar oder falsifizierbar. Sie ändern sich auch aufgrund der sich ändernden Wirklichkeit. 


\subsection{Wozu sind Stereotype da?}

Auch wenn Stereotype meist als ein nicht begrüßenswertes, gesellschaftliches Phänomen gelten, können ihnen mannigfache Funktionen nicht abgesprochen werden, die sie nolens volens zu einer - vor allem in sozialer Hinsicht - relevanten Erscheinung machen. Daher soll an dieser Stelle - um das Wesen von Stereotypen besser begreifen zu können - auch ihre Rolle diskutiert werden.

Quasthoff (1973: 40) ${ }^{4}$ unterscheidet zwischen folgenden Funktionen von Stereotypen:

- die kognitive Funktion, die es ermöglicht, zu reduzieren oder zu generalisieren und demzufolge die Wahrnehmung der Welt zu erleichtern,

- die soziale Funktion, die eine Grundlage für die Sicherung sozialer Zugehörigkeit bildet und den Prozess der Integration in die jeweilige Gruppe vereinfacht,

- die affektive Funktion, die die Erzeugung eines positiven Selbstbildes, Gemeinschaftsgefühls oder der Sicherheit bedingt.

Broschinsky und Schwabe (2011: 205) machen darauf aufmerksam, dass soziale Bedingungen zur Entstehung und Verbreitung von Stereotypen gegenüber Fremdgruppen einen Beitrag leisten. Denn sie ermöglichen es, die eigene Gruppe positiv von Fremden zu unterscheiden und demzufolge das Selbstbild aufzuwerten. Darüber hinaus können geplante oder bereits abgeschlossene Handlungen gegenüber Fremdgruppen gerechtfertigt werden, wobei diesen die Verantwortung für Missstände zugeschrieben wird. Auch unangenehme und unbeliebte Ereignisse sowie Entwicklungen im Rahmen der Gesellschaft können auf die Anwesenheit bzw. das Verhalten von fremden Gruppen zurückgeführt werden.

Die Aufgabe stereotypen Denkens besteht somit darin, das Funktionieren in der eigenen Gesellschaft (meist zuungunsten einer fremden Gruppe) weitgehend zu erleichtern bzw. überhaupt zu sichern:

Gesellschaften benötigen offensichtlich Stereotypen, um Gemeinsamkeit zu schaffen, und so werden oft genug auch Stereotypen direkt gefordert, oft genug bei der Beschreibung einer Bedrohung angesichts der alle Abwehrkräfte mobilisiert werden müssen (Hahn \& Hahn 2002: 36).

Im fremdsprachlichen Unterricht kommt Stereotypen eine wichtige Rolle $\mathrm{zu}^{6}$. Denn nach Keuten (2005: 182) seien sie ein lernprozessfördernder Anlass

\footnotetext{
${ }^{4}$ Vgl. Erll und Gymnich (2007) sowie Mikołajczyk (2002).

${ }^{5}$ Eine umfangreiche Charakteristik von Funktionen stereotypen Denkens liefert Konrad (2006: 132-140).

${ }^{6}$ Dazu siehe auch Löschmann (1998).
} 
in Bezug auf das interkulturelle Lernen ${ }^{7}$ und somit auf die Förderung von Empathiefähigkeit und die Entwicklung von Selbstbewusstsein. Insbesondere seien sie aber als Thema für mediengestützte Projekte geeignet, in denen Lernende ihre Kenntnisse über die zielsprachliche Kultur vertiefen könnten ${ }^{8}$.

Das Stereotyp als Forschungsgegenstand weckt das Interesse nicht weniger Wissenschaftler, wovon seine unzähligen Listen von Merkmalen oder Beschreibungen von Funktionen zeugen. Dies erleichtert allerdings die Auseinandersetzung mit diesem Phänomen nicht. Es macht sogar jegliche Herangehensweise an den Terminus noch komplizierter und undurchsichtiger. Nichtsdestotrotz lassen sich wissenschaftlich untermauerte Erwägungen auf einen gemeinsamen Nenner bringen: Stereotype gehören offensichtlich zum unverzichtbaren Bestandteil jeder Gesellschaft und beeinflussen die Beziehungen nicht nur im Rahmen einer einzigen Sprach- und Kulturgemeinschaft, sondern sie wirken sich auch auf jene zwischen den Vertretern unterschiedlicher sprachlich-kultureller Gemeinden auf. Da Stereotype unterschiedliche auch als durchaus negativ geltende - Funktionen erfüllen und damit in gewissem Sinne das Soziale (mit-)regeln, sind sie aus dem Alltag nicht wegzudenken. Daher ist zu überlegen, wie mit ihnen (auch im Fremdsprachenunterricht) umgegangen werden soll, um ihre Wirkung zumindest abzuschwächen.

\section{STEREOTYPE IM FREMDSPRACHENUNTERRICHT AM BEISPIEL EINES POLNISCH-DEUTSCHEN INTERNET-TANDEMPROJEKTS ${ }^{9}$}

\subsection{Projektrahmen}

Am polnisch-deutschen E-Mail-Projekt, das zwischen dem Institut für Germanistik der Adam-Mickiewicz-Universität in Poznań und der Fakultät für Linguistik und Literaturwissenschaft an der Universität Bielefeld im Wintersemester 2016/2017 durchgeführt wurde, nahmen insgesamt 14 Studierende im Bachelorstudium teil. Das Projekt wurde von Dr. Tina Claußen

${ }^{7}$ Aus Platzgründen wird im Beitrag auf das interkulturelle Lernen nicht näher eingegangen. Dazu siehe z.B. Bechtel (2003), Diel (2000), Loladze (2009), Mihułka (2010) sowie Sternecker und Treuheit (1993).

${ }^{8}$ Keuten (2005: 183) präsentiert auch ausgewählte Ideen für die Auseinandersetzung mit Stereotypen im Deutschunterricht. Hierzu gehören kreative Aufgaben zur Analyse von Autound Heterostereotypen anhand einer Fotosammlung oder ein auf deutsche Zeitschriften und Sprachlernmagazine für Schüler gestütztes Projekt, das die Darstellung von Polen und Deutschland in den genannten Publikationen in den Mittelpunkt stellt.

${ }^{9}$ Auf die Idee, Zielsetzungen, Teilnehmer, den Aufbau und Ablauf des Projekts sowie seine Evaluation aus der Sicht der Lehrenden und Lernenden wurde in Claußen und Pawłowska-Balcerska (2018) ausführlich eingegangen. 
(Bielefeld) und Dr. habil. Agnieszka Pawłowska-Balcerska (Poznań) koordiniert und dauerte insgesamt fünf Wochen.

Während alle Poznaner Probanden polnische Muttersprachler waren und zum Zeitpunkt der Projektdurchführung Germanistik als einziges Fach (5. Semester) studierten, bildeten ihre Bielefelder Partner, die sich für Deutsch als Fremdsprache im Haupt- oder Nebenfach entschieden, eine heterogene Gruppe in Bezug auf Erstsprachen, Herkunftsländer, Studienschwerpunkte und studierte Fächer.

Das Projekt wurde jeweils in eine Lehrveranstaltung integriert, in der fremdsprachliches Schreiben im Mittelpunkt stand. Es begann mit einer ersten Kontaktaufnahme der deutschen Probanden mit ihren polnischen Kommilitonen, denen im Vorfeld per Losverfahren Tandem-Partner zugeteilt wurden.

$\mathrm{Zu}$ den Zielen des gemeinsamen Vorhabens zählten:

- interkultureller Austausch zwischen Teilnehmern,

- Förderung der fremdsprachlichen Schreibfertigkeit auf der DaF-Fortgeschrittenenstufe,

- Ausprobieren ausgewählter Korrekturverfahren,

- Sammeln von Erfahrungen in Bezug auf Vorbereitung, Durchführung und Evaluation internationaler Projekte.

Am Anfang des polnisch-deutschen Projekts stand also der Wunsch, eine möglichst praxisbezogene / realitätsnahe Lehrveranstaltung anzustreben, die den Erwartungen der Poznaner und Bielefelder Probanden gerecht werden konnte. Die Bielefelder DaF-Studierenden waren für die polnischen Projektteilnehmer "echte“ Adressaten. Im Projekt konnte also ein authentischer Schreibanlass geschaffen werden, denn

Das Schreiben im Fremdsprachenunterricht wird ja auch deshalb oftmals als wenig motivierend empfunden, da Lernende "nur" für die Lehrenden schreiben, die die Texte dann bewerten und - zumindest in den Augen der Lernenden den Inhalten weniger Interesse entgegenbringen als der sprachlichen Realisierung (Claußen \& Pawłowska-Balcerska 2018: 660).

Die deutschen Probanden konnten auch die in ihrem Seminar kennengelernten Korrekturverfahren ausprobieren und reflektieren.

\subsection{Stereotype aus dem Blickwinkel polnischer und deutscher eTandemprojekt-Teilnehmer}

Als Ausgangspunkt der sprachlich-interkulturellen Auseinandersetzung mit schriftlichen Produktionen galten Texte mit dem Thema "So sehe ich meine Landsleute", die, von polnischen Studierenden verfasst, an ihre deutschen Kommilitonen verschickt wurden. 
Die Aufgabenstellung sollte zur Reflexion über sich selbst und über andere bewegen. Deckers (2010: 524) zufolge bestehe die Notwendigkeit, Lernenden die Erkenntnis der eigenen kulturellen Prägung in der Begegnung mit anderen Kulturen möglich zu machen, um sie auf diese Weise zur Entwicklung einer reflektierten Sichtweise zu befähigen. Denn

[e]rst wenn eigenkulturelle und eigensprachliche Erfahrungen im Fremdsprachenunterricht betrachtet werden, können sich Lernende vertiefend über interkulturelle Themen austauschen und einander von ihren subjektiven Bildern der eigenen und fremder Kulturen erzählen. Diese subjektiven Bilder sind oft Stereotype, die aus dem Fremdsprachenunterricht kaum ausgeschlossen werden können (Marques-Schäfer, Bolacio Filho \& Stanke 2016: 568).

Im Fremdsprachenunterricht sollte es allerdings auf gar keinen Fall darauf ankommen, kulturelle Unterschiede bloß zu nennen und $\mathrm{zu}$ memorieren. Diese Vorgehensweise würde nämlich zum Verfestigen von Verhaltenserwartungen und Stereotypen führen (Mihułka 2010: 56). Stereotype selbst sollen eher als Grundlage für das Betrachten des Ich bzw. Wir vor dem Hintergrund des Fremden (selbstverständlich auch umgekehrt) gelten und auch das Entdecken von Gemeinsamkeiten erleichtern. Sich auf die Suche nach Differenzen zwischen den Vertretern verschiedener Sprach- und Kulturgemeinschaften zu beschränken, wäre sicherlich ein grobes Versäumnis. Stereotype sollten zum Verständnis des Anderen und zugleich des Eigenen einen Beitrag leisten. Dies ist allerdings erst dann möglich, wenn man Fremdsprachenlernende für das Bestehen anderer Sprach- und Kulturhorizonte sensibilisiert.

Die Texte (Umfang ca. 300 Wörter) wurden zum Auslöser einer regen E-Mail-Korrespondenz über Auto- und Heterostereotype ${ }^{10}$ und insbesondere über deren Allgegenwärtigkeit, Provenienz, Wesen und Wahrheitsgehalt. Die schriftlichen Aussagen wurden von der Bielefelder Gruppe einer Korrektur unterzogen.

Auf die eingangs erwähnte allgemeine Präsenz von Stereotypen wurde von den polnischen und deutschen Studierenden mehrmals eingegangen:

Text-Passage 1: Es gibt natürlich viele allgemeine Stereotype über Polen - wie über alle anderen Länder ${ }^{11}$.

Text-Passage 2: Heutzutage, wo die Grenzen offen sind, können die Menschen frei in einen fremden Land fahren. Sie haben ständig Kontakt mit den Auslän-

${ }^{10} \mathrm{Zu}$ nationalen Stereotypen siehe beispielsweise Erll und Gymnich (2007), Hahn und Hahn (2002), Mikołajczyk (2002) und Wowro (2010).

11 Alle Text- bzw. E-Mail-Passagen wurden in diesem Beitrag im Originalwortlaut übernommen. 
der. Jedes Land ist charakteristisch und hat etwas, was es von anderen unterscheidet. Polen ist auch auf seine Art charakteristisch. Es gibt auch verschiedene Stereotypen über Polen.

Text-Passage 3: Jedem Land werden Stereotypen zugeschrieben.

In den studentischen Aussagen wurde auch der verallgemeinernde Charakter von Stereotypen im Sinne von Berting und Villain-Gandossi (1999: 14-15), Hahn und Hahn (2002: 22) sowie Quasthoff (1973: 28 und 40) betont, wobei meistens vor Generalisierungen in Bezug auf andere Nationen gewarnt wurde:

E-Mail-Passage 1: Es ist schwer, ein ganzes Land auf ein paar Eigenschaften und Besonderheiten zu reduzieren.

Text-Passage 4: Die Polen unterscheiden sich voneinander. Sie sind „keine mentale Einheit" - ihre Denkweise wird durch ihren Wohnort, ihre Region geprägt.

In den Texten der polnischen Studierenden wurden viele gängige Stereotype über Polen (wie z.B. (Auto-)Diebstahl oder hoher Alkoholkonsum) thematisiert:

Text-Passage 5: Viele Ausländer glauben, dass Polen sehr arm sind. Sie behaupten, dass Polen sehr armes Land ist. Das stimmt nicht. Polen ist armer als zum Beispiel Deutschland, aber auch reicher als zB. die Ukraine. Polen in großen Städten leben sehr schnell. Sie haben immer viel zu tun und sind immer in Zeitdruck. Was Trinken angeht, trinken Polen viel Kaffee. Sie trinken Kaffee jeden Morgen und auch nachmittags. Am öftesten trinken sie einen Milchkaffee. Verschiedene Ausländer behaupten, dass Polen viel Alkohol, viel Wodka trinken. Das stimmt, dass Alkoholkonsum in Polen groß ist, aber das muss nicht heißen, dass jeder Pole ein Alkoholiker ist. [...] Auf jeden Fall, sind Polen sehr gastfreundlich und umtriebig. Polen sind auch traditionsbewusst. Die Tradition ist für sie sehr wichtig.

Interessant ist dabei, dass die Poznaner Studierenden neben positiven auch viele negative Stereotype nannten, obschon sie sich zugleich bemühten - wie im Text oben - Polen vor dem Hintergrund anderer Nationen zu betrachten und demzufolge Vergleiche mit ihnen zu wagen. Die Antwort des Bielefelder Studierenden auf den bereits präsentierten Text zeugt eindeutig davon, dass stereotypes Denken des Öfteren - wie im theoretischen Teil hervorgehoben wurde ${ }^{12}$ - die Übernahme von weit verbreiteten Aussagen Dritter bedeutet und sich nicht auf die eigene Erfahrung stützt:

${ }_{12}$ Dazu siehe im theoretischen Teil des Beitrags Berting und Villain-Gandossi (1999: 14-15), Hahn und Hahn (2002: 22), Pfeiffer (2000: 127), Six (1987: 44) und Telus (2002: 66-67). 
E-Mail-Passage 2: Alles was genannt wurde, sind nur Stereotype. Die Menschen bilden sich Meinungen über andere Menschen ohne sie zu kennen und auf Grundlage von Geschichten, welche sie gehört haben.

E-Mail-Passage 3: Ich selbst war ein Mal ganz kurz, für ungefähr ein oder zwei Stunden in Polen, da konnte ich nicht so viele Erfahrungen sammeln. Also weiß ich alles, was ich über "die Polen“ weiß nur aus den Erfahrungen von anderen oder Polen, die hier in Deutschland leben. Dass Polen viel Alkohol trinken ist hier ein recht geläufiges Stereotyp, dass sie viel Kaffee trinken ist mir allerdings neu. Aber es ist ja auc viel interessanter zu erzählen, dass andere viel Alkohol trinken, Kaffee ist ja langweilig. Wie das immer so ist. [...] Interessant finde ich auch, dass du schreibst, dass viele meinen Polen wäre ein sehr armes Land, das war mir bisher gar nicht so bewusst. Wenn ich drüber nachdenke fällt mir aber ein, dass eine Freundin meiner Mama erzählt hat, dass sie sich mal in einer polnischen Stadt verlaufen hat und dann in einem besonders armen Viertel war, die Menschen dort haben wohl teilweise in Blechhütten gewohnt, das fand ich schon ziemlich heftig, vor allem weil es eigentlich ein touristischer Ort war und der größte Teil des Ortes wohl sehr schön hergerichtet war

Text-Passage 6: Allgemein kann ich sagen, dass manche Stereotype aus Unwissenheit über das Land oder den falsch gehörten Informationen folgen.

Auch in einem anderen Text wurden sowohl positive als auch negative Eigenschaften von Polen diskutiert, wobei der Studierende diesmal auch die deutsche Perspektive einzunehmen versuchte:

Text-Passage 7: Der stereotypische Pole ist fleißig, aber er verbringt gern die Freizeit mit Alkohol. Der stereotypische Pole hat seine Vorteile und Nachteile, wie jede Person. Im Westen von Polen kann man sich sehr oft mit der Meinung treffen, dass der Pole ein Schwindler und ein Dieb ist, aber zum Glück ändern immer mehr Menschen ihre Meinung. Heutzutage, wenn wir z.B. den Deutschen nach der Meinung über die Polen fragen, antwortet er, dass er schon einige Polen kennt (wahrscheinlich aus der Arbeit) und sie sind fleißig, hilfreich und herzlich. Die Ausländer vergessen nicht die Polinnen. Die Frauen sind schön, liebevoll und können sich gut um die Nestwärme kümmern.

Die im Text aufgegriffenen Stereotype wurden von der deutschen Seite bestätigt, da sie in Deutschland sehr verbreitet sind. Darüber hinaus wurden sie dem Bild von der eigenen Nation gezielt gegenübergestellt, um auch Gemeinsamkeiten zwischen beiden zu finden ${ }^{13}$ :

E-Mail-Passage 4: Ich bin sehr begeistert von deinem Text und finde diesen sehr interessant. Besonders gefällt mir, dass du am Anfang direkt auf die wohl bekanntesten Stereotypen eingegangen bist, welche die meisten von uns kennen.

13 Dazu siehe Mihułka (2010: 56). 
Zum Beispiel habe ich mit Polen das typische Klischee des Diebstahls assoziiert, welches bei vielen Menschen hier sehr bekannt ist. Außerdem war mir ebenfalls bekannt, dass Polen und Polinnen gerne viel trinken. Mittlerweile glaube ich aber, dass es bei den Deutschen nicht anders ist.

Sehr überraschend finde ich, wie genau du das Bild der Deutschen von polnischen Landsleuten beschreibst. Denn ich persönlich finde auch, dass die Polen und Polinnen, die ich kenne, immer sehr fleißig, hilfreich und herzlich sind. Ebenso ist mir auch bekannt, dass polnische Frauen immer als sehr schön und ansehnlich empfunden werden.

Die Deutschen waren allerdings nicht nur an den geläufigen Stereotypen über Polen bzw. über die eigenen Landsleute interessiert. Sie baten ihre Tandempartner nicht selten auch darum, zum eigenen Text Stellung zu nehmen:

E-Mail-Passage 5: Meine einzige Frage zum Inhalt wäre: Was hältst du von diesen Klischees? Kannst du einigen zustimmen oder überhaupt nicht?

Auf die oben gestellte Frage folgte eine (selbst-)kritische, distanzierte Antwort des polnischen Studierenden, der sich keinesfalls um das Verbessern des Bildes von der eigenen Nation bemühte:

E-Mail-Passage 6: In deinem Feedback fragst du mich, was ich von den Klischees finde. Es tut mir Leid, aber ich verstehe die Stereotype von Polen als Dieb. Früher war es wirklich so, dass Polen in Deutschland gestohlen haben. Jetzt glaube ich nicht so oft und intensiv. Kennst du den Witz: "Kommen Sie nach Polen, ihr Auto ist schon da"? ;)

Man sagt auch, dass man in Polen viel trinkt. Ok, ich stimme damit zu, aber man trinkt nicht so viel wie in Russland oder in der Ukraine ;) Ich persönlich trinke nur einen Sekt zum Silvester. Meine Familie trinkt auch selten. Ich finde, dass der Alkoholverbrauch sich in den letzten Jahren in Polen verringerte.

Wenn es um die hübschen Frauen geht, stimme ich nicht damit zu. Letzte Sommerferien verbrachte ich in Konstanz. Unglaublich, wie viele attraktive Frauen habe ich dort gesehen.

Trotz vieler Stereotype, denen die eine oder andere Seite zustimmte, wurde des Öfteren der Versuch unternommen, sie zu relativieren und zugleich ihren subjektiven Charakter zum Ausdruck zu bringen. Dies kann sicherlich als ein wichtiger, erfreulicher - wenn auch nur kleiner - Schritt in Richtung Abbau von Stereotypen angesehen werden:

E-Mail-Passage 7: Bevor man über Personen urteilt, sollte man sich selbst erst davon überzeugen ob es auch der Wahrheit entspricht.

Text-Passage 8: Ich empfehle jeder Person den Text in Wirklichkeit in Polen konfrontieren. 
Text-Passage 9: Darum lade ich jede Person ein, die nur unsere Staat kennen lernen will, zum Besuch des Großpolens, damit sie selbst dafür entscheiden konnte, wie wir wirklich sind.

Die Studierenden wussten das Thema "So sehe ich meine Landsleute" zu schätzen, was auch in der das Projekt abschließenden schriftlichen Befragung zum Ausdruck gebracht wurde:

Passage 1 (schriftliche Befragung): Das Thema dieses Schreibprojekts an sich finde ich aber sehr interessant. Stereotype gibt es in jedem Land und sie haben mit der Wahrheit oft nicht viel zu tun. Deswegen auch bin ich der Meinung, dass man darüber diskutieren sollte.

Ein Bielefelder Projektteilnehmer äußerte auch den Wunsch, auf die im Projekt thematisierten Stereotype noch detaillierter eingehen $\mathrm{zu}$ wollen. Denn er würde gern wissen,

Passage 2 (schriftliche Befragung): Wie die Stereotype (die ja alle nicht der Wahrheit entsprechen) entstanden sind.

Es bestand somit unter den DaF-Studierenden reges Interesse an Stereotypen als einem sozialen Phänomen und zugleich die Notwendigkeit sie zu reflektieren. Daher wäre zu überlegen, wie ein separates Seminar zu diesem Thema gestaltet werden sollte, in das ein E-Mail-Tandem-Projekt integriert werden könnte.

\section{SCHLUSSFOLGERUNGEN}

Dass Stereotype kein neues und oft lange unveränderbares, soziales Phänomen sind, wurde an mehreren Stellen des vorliegenden Beitrags betont. Nichtsdestotrotz oder gerade deshalb ist es von hoher Relevanz, sie nicht nur auf der Ebene unterschiedlicher Fachdisziplinen zu diskutieren. Sie sollen auch im institutionalisierten Schulwesen zur Sprache gebracht werden.

Hierzu scheint sich der fremdsprachliche Unterricht besonders gut $\mathrm{zu}$ eignen, da seine Aufgaben nicht ausschließlich in der Vermittlung von Wortschatz oder Grammatik oder in der Entwicklung der vier Sprachfertigkeiten zu sehen sind. Auch die Sensibilisierung für das Neue, Andere, Unbekannte und zugleich für das Eigene genauso wie die Förderung von Toleranz gegenüber dem Fremden und demzufolge ein kleinschrittiger Abbau von Stereotypen gehören zu seinen Pflichten und können zur Förderung der 
interkulturellen Kompetenz ${ }^{14}$ einen Beitrag leisten, die wiederum einem effizienten Umgang mit Mitgliedern anderer Sprach- und Kulturgemeinschaften zugrunde liegt.

Am Beispiel des polnisch-deutschen E-Mail-Tandemprojekts war man bemüht, zu zeigen, dass der Umgang mit Stereotypen kein bloßes Auflisten von mehr oder weniger verbreiteten Auto- und Heterostereotypen sowie keine strikte Trennung in Ich / Wir und Ihr / Sie bedeuten kann. Im Fremdsprachenunterricht ist es möglich, die Auseinandersetzung mit stereotypen Vorstellungen in die Wege zu leiten, indem man die Vertreter einer anderen Sprach- und Kulturgemeinschaft zum Dialog einlädt und sie ihre eigene Sichtweise präsentieren lässt. Dann gelten Stereotype nicht mehr als eine (nicht selten kritiklos und unreflektiert übernommene) Selbstverständlichkeit, sondern sie erweisen sich als eine höchst komplexe, allgemein präsente, des Öfteren die Wirklichkeit verzerrende und sozial gerechtfertigte Erscheinung, über die man diskutieren soll und kann. Im interkulturellen Dialog ist allerdings die Rücksicht auf das Gegenüber und somit auf sein Recht auf die Andersartigkeit das höchste Gebot.

\section{LITERATURVERZEICHNIS}

Adamczak-Krysztofowicz, S. (2005). Kooperatives Miteinander statt Nebeneinander. Zur Beziehung zwischen der interkulturellen Fremdsprachendidaktik und den Kulturwissenschaften. Glottodidactica, XXX/XXXI, 5-11.

Bartmiński, J. (1999). Unsere Nachbarn aus der Sicht der Studenten (Aus Untersuchungen über nationale Stereotypen). In: T. Walas (Hrsg.), Stereotypen und Nationen (S. 311-323). Krakau: Internationales Kulturzentrum.

Bechtel, M. (2003). Interkulturelles Lernen beim Sprachenlernen im Tandem. Tübingen: Gunter Narr Verlag.

Berting, J. / Villain-Gandossi, Ch. (1999). Rolle und Bedeutung von nationalen Stereotypen in internationalen Beziehungen: ein interdisziplinärer Ansatz. In: T. Walas (Hrsg.), Stereotypen und Nationen (S. 13-31). Krakau: Internationales Kulturzentrum.

Broszinsky-Schwabe, E. (2011). Interkulturelle Kommunikation Missverständnisse - Verständigung. Wiesbaden: VS Verlag für Sozialwissenschaften.

Claußen, T. / Pawłowska-Balcerska, A. (2018). Internationale Lehrkooperationen am Beispiel eines deutsch-polnischen E-Mail-Tandems mit fortgeschrittenen Deutschlernenden und angehenden DaF-Lehrenden. Info DaF, 5, 655-671.

Dąbrowska, J. (1999). Stereotype und ihr sprachlicher Ausdruck im Polenbild der deutschen Presse: eine textlinguistische Untersuchung. Tübingen: Narr.

${ }^{14}$ Aus Platzgründen wird im vorliegenden Beitrag auf die interkulturelle Kompetenz nicht näher eingegangen. Dazu siehe u.a. Adamczak-Krysztofowicz (2005), Pawłowska (2014), Pfeiffer (2000) und Pieklarz (2006). 
Deckers, M. (2010). Im Kulturkontakt gebildete Stereotype als Teil eines kulturellen Lernprozesses - untersucht in den Weblogs von in Deutschland lebenden Amerikanern. Info DaF, 6, $521-545$.

Diel, D. (2000). Interkulturelles Lernen: Auf dem Weg zum gegenseitigen Verständnis und Respekt. In: K. Götz (Hrsg.), Interkulturelles Lernen/Interkulturelles Training (S. 221-234). München: Rainer Hampp Verlag.

Erll, A. / Gymnich, M. (2007). Interkulturelle Kompetenzen - Erfolgreich kommunizieren zwischen den Kulturen. Stuttgart: Klett.

Hahn, H. H. / Hahn, E. (2002). Nationale Stereotypen. Plädoyer für eine historische Stereotypenforschung. In: H. H. Hahn (Hrsg.), Stereotyp, Identität und Geschichte. Die Funktion von Stereotypen in gesellschaftlichen Diskursen (S. 17-56). Frankfurt am Main / Berlin / Bern: Peter Lang.

Keller, G. (1969). Die Funktion von Stereotypen beim Erkenntnisprozess im kulturkundlichen Unterricht - dargestellt an einer Strukturanalyse von Schülerurteilen. Die Neueren Sprachen, 68, 175-186.

Keuten, H.-P. (2005). Stereotype, interkulturelles Lernen und konstruktivistische Fremdsprachendidaktik. Didaktische Überlegungen und Workshopbericht. In: A. Kowalczyk / J. Pacholski (Hrsg.), Stereotype in interkultureller Wahrnehmung (S. 178-187). Nysa: Oficyna Wydawnicza Państwowej Szkoły Zawodowej.

Koch, A. (2002). DruckBilder: Stereotype und Geschlechtercodes in den antipolnischen Diskursen der "Gartenglaube" (1870-1930). Köln: Böhlau.

Konrad, J. (2006). Stereotype in Dynamik. Zur kulturwissenschaftlichen Verortung eines theoretischen Konzepts. Tönning / Lübeck / Marburg: Der Andere Verlag.

Lippman, W. (1944) (orig. 1922). Public opinion. New York: Macmillan.

Loladze, N. (2009). Interkulturelles Lernen als Schlüsselqualifikation im Zeitalter der Globalisierung im Kontext des Deutschunterrichts in Georgien. Glottodidactica, XXXV, 151-161.

Löschmann, M. (1998). Stereotype, Stereotype und kein Ende. In: M. Löschmann, M. Stroinska (Hrsg.), Stereotype im Fremdsprachenunterricht (S. 7-33). Frankfurt am Main / Berlin / Bern: Peter Lang.

Marques-Schäfer, G. / Bolacio Filho, E. / Stanke Sol. R. (2016). Was können Lehrwerke zur Reflexion von Stereotypen im DaF-Unterricht beitragen? Eine Analyse anhand der Arbeit mit DaF kompakt in Brasilien. Info DaF, 5, 566-586.

Mihułka, K. (2010). Stereotype und Vorurteile in der deutsch-polnischen Wahrnehmung. Rzeszów: Wydawnictwo Uniwersytetu Rzeszowskiego.

Mikołajczyk, B. (2002). Deutschlandbilder in der polnischen EU-Beitrittsdebatte über die persuasive Leistung nationaler Heterostereotypen in der politischen Rede. Scripta Neophilologica Posnaniensia, IV, 61-85.

Pawłowska, A. (2014). (Un-)typisch deutsch? (Un-)typisch polnisch? - Polnische Germanistikstudierende über sich selbst und ihren deutschen Nachbarn. Studia Germanica Gedanensia, 31, 266-277.

Pfeiffer, W. (2000). Möglichkeiten und Grenzen der interkulturellen Sprachvermittlung. Glottodidactica, XXVIII, 125-139.

Pieklarz, M. (2006). Stereotype und Affektivität im interkulturellen Fremdsprachenunterricht. Glottodidactica, XXXII, 109-121.

O'Sullivan, E. (1992). Aktuelles Fachlexikon. Stereotyp und Vorurteil, Selbstbild und Fremdbild. Fremdsprache Deutsch, 6, 65.

Quasthoff, U. (1973). Soziales Urteil und Kommunikation. Eine sprachwissenschaftliche Analyse des Stereotyps. Frankfurt am Main: Athenäum Fischer Verlag. 
Schaff, A. (1980). Stereotypen und menschliches Handeln. Wien: Europaverlag.

Six, B. (1987). Stereotype und Vorurteile im Kontext sozialpsychologischer Forschung. In: G. Blaicher (Hrsg.), Erstarrtes Denken. Studien zu Klischee, Stereotyp und Vorurteil in englischsprachiger Literatur (S. 41-54). Tübingen: Narr.

Sternecker, P. / Treuheit, W. (1994). Ansätze interkulturellen Lernens. In: H. Otten / W. Treuheit (Hrsg.), Interkulturelles Lernen in Theorie und Praxis (S. 31-56). Opladen: Leske + Budrich.

Telus, M. (2002). Gruppenspezifisches Stereotyp: ein Modell der Einbettung in die Textproduktion an russischem, polnischem und deutschem Material. Frankfurt am Main: Peter Lang.

Wowro, I. (2010). Stereotype aus linguistischer und didaktischer Sicht. Stereotypisierungen in ausgewählten Lehrwerken für DaF. In: M. Grimberg / S. H. Kaszyński (Hrsg.), Convivium. Germanistisches Jahrbuch Polen (S. 303-325). Bonn: Deutscher Akademischer Austauschdienst.

Received: 26.09.2018; revised: 8.07.2019 